\title{
QoS and Performance Analysis using VOLTE in 4G Mobile Network
}

\author{
H. P. Bramhaprakash \\ Department of Computer Science \& Engineering \\ Sri Siddhartha Institute of Technology,Tumkur, India \\ Dr.MANJAIAH D H \\ Dept of computer science \\ Mangalore university, Mangalore,India
}

\begin{abstract}
Voice over LTE or VoLTE is a simple and effective solution for voice in an LTE network. We are going to discuss about the features, functionalities and techniques that makes VoLTE [1] the best suited solution for providing better Quality of Service to the 4G subscribers. In this paper, we show that the VoLTE is equally better as VoIP technology which is used worldwide by millions of smart phone users for data and voice calls through 4G.

Categories and subject descriptors

[Computer Communication Networks]: 4G communications, All-IP communication networks;
\end{abstract}

General Terms - VoLTE, 4G, quality of service (QoS), design;

Keywords - Cellular networks; voice; VoLTE*; 4G;

\section{INTRODUCTION}

At present stage voice is exceptionally straightforward however effective arrangement of correspondence for some cell phone clients on the planet. From the introduction of cell phones the voice administrations gave to the clients that ensured the nature of administration (QoS) for voice calls has been popular till date. Voice calling administrations to the clients have customarily been bolstered by the Circuit-Switched (CS) arrange, where a committed circuit or channel is to be built up to transmit voice over the movement. Presently a-days this innovation got to be distinctly invalid as the system advances are progressed toward the Long Term development (LTE) and fourth era (4G) mechanical norms where Packet Switched (PS) systems are executed. This relocation of system advancements is the consequence of the nuts and bolts irreversible change that happened in the engineering of LTE.

Voice over LTE (VoLTE) was designed to fulfill this purpose of migration in technologies, whose straightforward design carries the voice over IP-based LTE network in packets not by a dedicated circuit in CS network technology. The voice communications maintains sessions for signaling separately for each calls to provide VoLTE voice calls. VOLTE has unlocked the feature of all-IP communications in the $4 \mathrm{G}$ network.

With the assistance of VoLTE the suppliers can:

- Provide the customers the brand new 4G LTE network and simultaneous delivery of data with crisp HD quality of voice.

- Collaborating different features like video calling and voice calling a new blend in calling can be served which attract the customers to opt for the particular service providers from the market.

- To inspire the customers with more and more conversations and converging them to be carried out by the single media rather using different media of communications.

- The administrators have more advantages and lower dangers, as administrators give voice calls and can include video, informing and converge with web by Web Real Time Communication (WebRTC) [15]. 
The operators are efficient enough to associate with different application providers to provide the users with best experience they can have at the lowest cost. They can also mainstream the innovations of the application developer in the communication services. This technology enables the provider to experiment with new features in the Long Term Evolution model regardless of the fact where the experiment is taking the company to in near future. VoLTE is an innovation that is based upon all-IP correspondence that furnishes $4 \mathrm{G}$ arrange clients with more limit with higher data transmission, bringing down dormancy and with new evaluating less expensive than the already existing systems in the market.

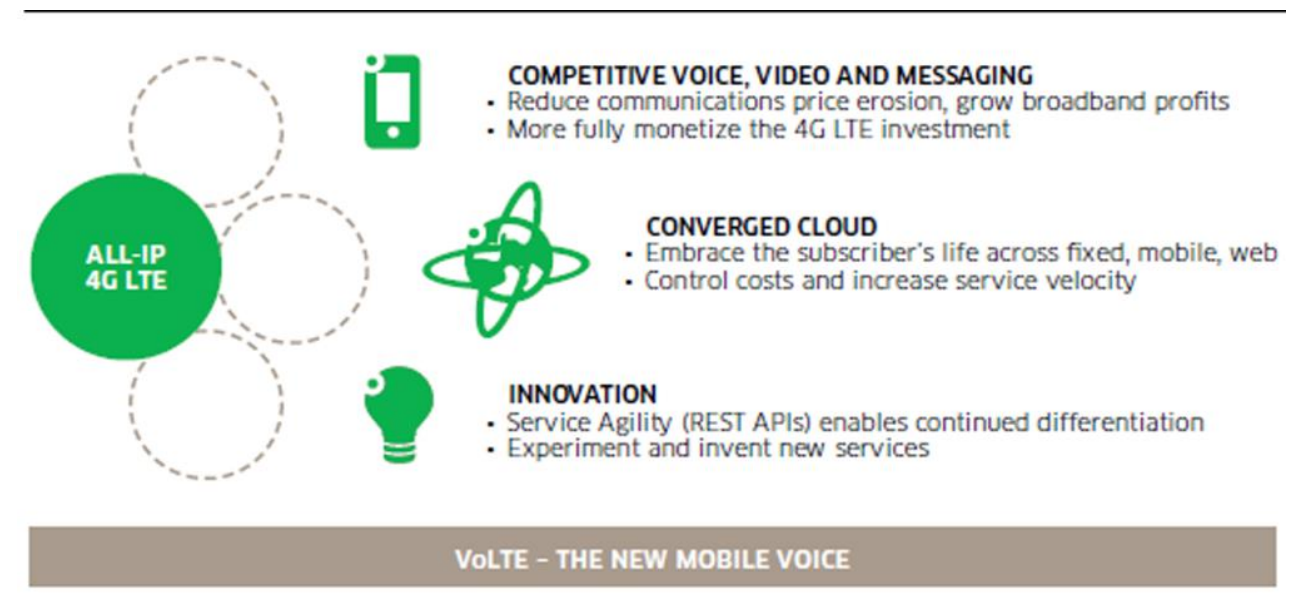

Figure 1: The all-IP communication network in 4G LTE.

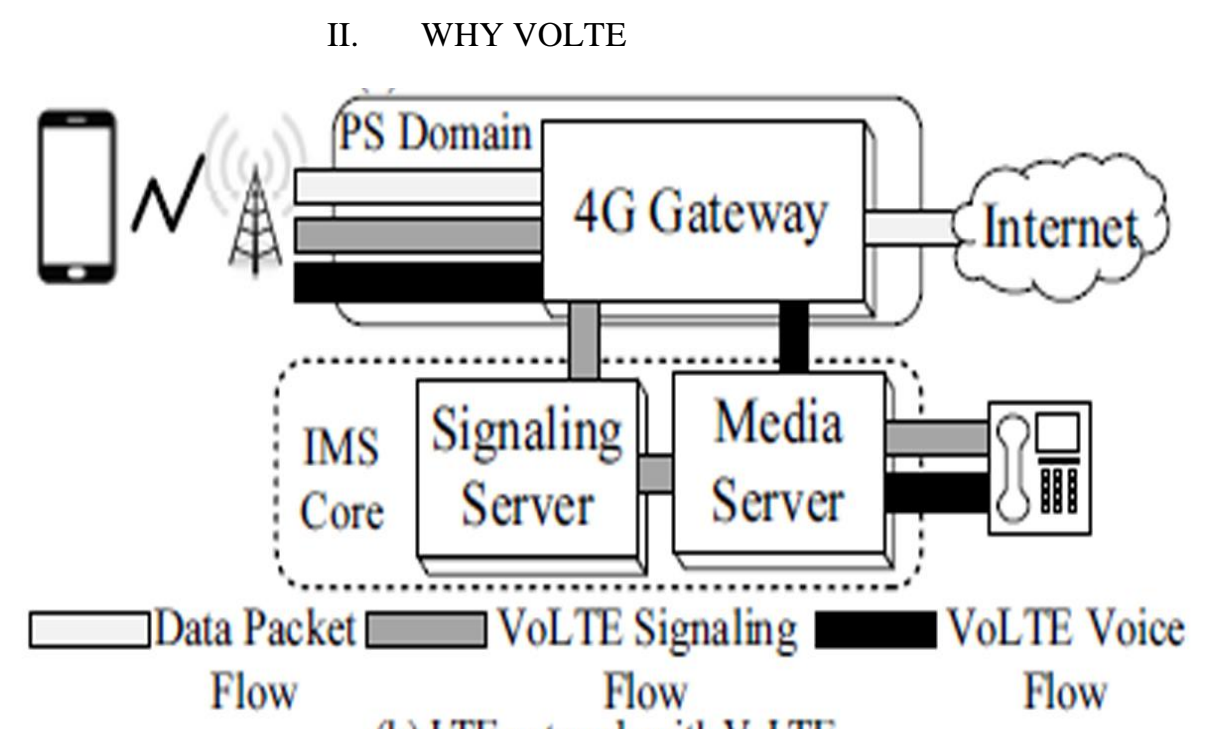

(b) LTE network with VoLTE

Figure 2: LTE organize engineering with VoLTE.

The voice over LTE is intended to travel from the already utilized circuit-exchanged (CS) to the bundle exchanged (PS) organize which is utilized as a part of the present system models. As we probably am aware every calls of VOLTE needs to keep up two distinctive and separate sessions for every calls, i.e. one for the information and other for the control plane. The control-plane is in charge of the message trade of the call flagging by means of Session 
Initiation Protocol or (SIP) [5] and the information plane is in charge of conveying the voice through Internet Realtime Transport Protocol or (RTP) [6]. There are two subsystems of the LTE arrange which are conjured in the VoLTE operations. To start with is the IP Multimedia Subsystem (IMS) center which is intended to help bolster the all-IP communication and sight and sound administrations [2]. It contains two segments; one is the media entryway and other is the VoLTE server. The media entryway is mindful to give continuous interactive media, for example, voice or video to the $4 \mathrm{G}$ clients or the phone clients. The VoLTE servers are to offer elements of the session control to the media gadgets. The IMS center in VoLTE is not limited to convey just to the VoLTE benefits additionally can be utilized to help bolster more propelled administrations sooner rather than later.

The second subsystem is the parcel exchanged (PS) whose significant segment is the $4 \mathrm{G}$ entryway which is like the edge directing of the web framework. Its primary capacity is to build up PS association among the cell phone and the administrator. It can likewise be utilized as a part of giving different administrations like-bundle separating, charging support, IP address distribution and approach requirement.

\section{FACTORS}

\section{A. DEPLOYMENT AND OPERATION COST}

Sending of the VoLTE can be accomplished by two objectives. One is by voice calls between the VoLTE supporters and other by building up calls among one of the VoLTE client and a conventional client. In this manner, the cost is charged upon two unique sources. Presently, the administrator needs to utilize another IMS center and needs to adjust the current subsystem. The PS space and the OAM (Operation, Administration and Maintenance) [7] are the current subsystems in the $4 \mathrm{G}$ arrange design that are should have been reconfigured. The CS space exhibit in the outline is likewise should have been overhauled which will bolster the SRVCC [8] to exchange the client from the $4 \mathrm{G}$ administrations to the $3 \mathrm{G}$ or $2 \mathrm{G}$ benefit once the client outbound the of $4 \mathrm{G}$ administration territory. The IMS center sent amid the VoLTE calls communicate with cell phones and customary communication framework to help move from the VoLTE signs to the CS based configuration.

\section{B. COMPARABLE CALL PERFORMANCE}

We analyze the VoIP and the VoLTE administrations based upon call setup time, call drop rate, and voice execution. In our trial we include 50 individuals at 10 distinct areas with 20 switches for versatility. Presently, we look at their call execution based upon three viewpoints:

a. Call setup time: we demonstrate the call setup time in four distinct situations, (a) VoLTE-TO-VoLTE (b) HANGOUT-TO-HANGOUT (c) VoLTE-TO-CS (d) HANGOUT-TO-CS. Here, in our experiment A $\rightarrow \mathrm{B}$ means callers with the technology A make calls to the person having call technology B. we now intercept both the calls with the same technology. The beneath figure plots the information blocked in two territories, one with frail flag and other with solid flag.

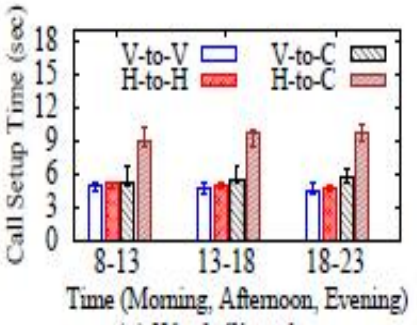

(a) Weak Signal

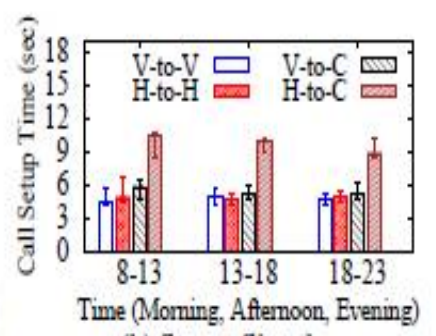

(b) Strong Signal

Figure 4: call setup time (most extreme, medium, least) in powerless flag and solid flag territories. 
In situation (1) and (2) the middle esteem is 5.0 seconds with contrast littler than $10 \%$ in three various types of flag quality. While in situation (3) and (4) it requires 3.7-4.8 seconds a greater amount of the middle esteem. Accordingly the VoLTE organize in $4 \mathrm{G}$ has a superior execution in contrast with the home base servers.

b. Voice quality: we carry out our experiment to compare the call quality or voice quality by sending an audio from person with technology A and send it to the person with technology B to receive it. We use noise suppression technique to prevent the background noises being recorded. The audio is again sent back to the user with the help of an audio line connected through the computer and then it is forwarded to another computer by a different audio line. Then the voice is recorded by software named AUDACITY [10] in 32-bit, $44.1 \mathrm{KHz}$ format.

We consider five particular settings: (a) both the gadgets are in a solid flag range (b) both the gadgets are in frail flag territory (c) the recipient is in a swarmed zone however having solid flag quality (d) the collector is in versatility (e) the sender is having a CS-based gadget and the beneficiary is in solid flag zone. We direct our analysis in view of the strategies and settings plotted by ITU [9].

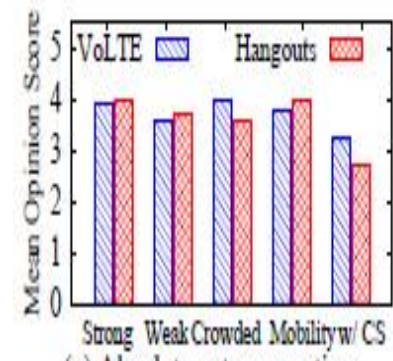

(a) Absolute category rating

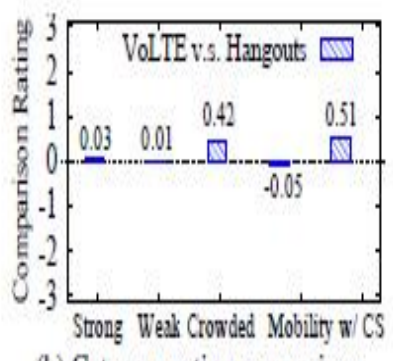

(b) Category rating comparison

Figure 5: Voice quality examination amongst VoLTE and Hangout.

The given figure plots the normal ACR score for Hangout and VoLTE in five particular situations. In the principal, second and fourth case the distinction is beneath 0.2 and is tantamount. In third and fifth case the execution of VoLTE is superior to home base by 0.4 in the swarmed districts, which improves it than others.

c. Call drop rate: we have noticed in our experiment that the rate of call drop in static situation is comparable and is negligible in VoLTE and VoIP.

But in the mobile environment when there is no inter-system switch the VoIP drop calls by a rate of $0.5 \%$ and VoLTE in other hand does not drop calls in this scenario. But, when the inter-system switch occur the ratio of call drops increases from $0.5 \%$ and $0 \%$ to $4 \%$ and $8 \%$ respectively for VoIP AND VoLTE [1]. From this we can conclude that VoLTE has an increased call drop rate than the VoIP network in inter-system switch.

\section{VOLTE DESIGN}

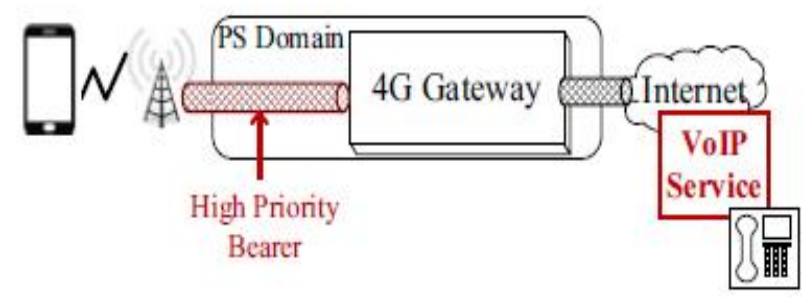

Volume 8 Issue 1 January 2017 
In here, we discuss about the design of VoLTE, which becomes the best among all other network infrastructures by the usage of VoIP schemes of internet. By using LTE in 4G both the subscribers and the service providers can benefit from it. The subscriber gets to use the services like good quality of calls with many different options and at cheaper rate. The service providers get the benefit of prioritized service delivery from the LTE networks. The VoLTE services are adopted by only those operators who are able to afford for establishing, deploying and maintaining these costly services for their customers.

\section{HOW IT WORKS}

The 4G LTE design comprises of developed Universal Terrestrial Radio Access Network (eUTRAN) and Evolved Packet Core (EPC). The 4G arrange utilizes the carrier idea to course IP movement from UE to the PDN. The carrier in system is an information session of IP having a characterized of QoS. A default conveyor is built up when a UE is joined to a 4G system and after that discharged when the system is set up by the application. These bearers utilized as a part of setting up the system are called committed bearers.

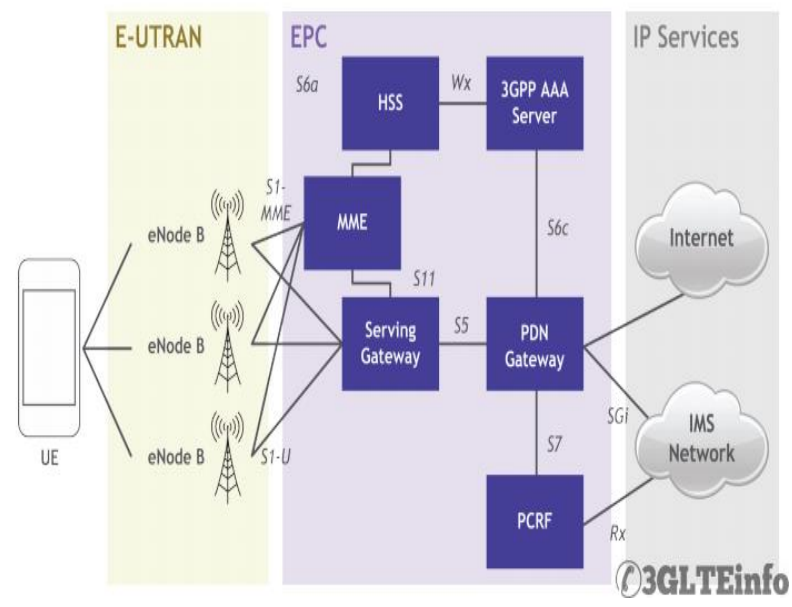

Figure 7: 4G LTE network architecture.

The components of 4G LTE network architecture are described below:

- UE: it is the device that user uses to connect to the $4 \mathrm{G}$ network such as, laptop, mobile, tablet etc.

- Evolved hub B (eNodeB): it is radio system engineering dissimilar to the layered design of $3 \mathrm{~g}$ which can improve the Radio Access Network's (RAN'S) operations.

- Serving Gateway (SG): all bundles like flagging and carrier goes through the serving entryway, which is a nearby portable stay for clients when they go in various eNodeBs or in spots of legacy $2 \mathrm{G}$ or $3 \mathrm{G}$ arrange regions.

- PDN Gateway: the PDN Gateway (P-GW) gives administration administrations to IP address, stream based arrangement for charges that are chosen by the administrator and QoS requirements as indicated by the approach when they get from the Policy control and Charging Rules Function (PCRF).

- PDN: the PDN includes network connection to $4 \mathrm{G}$ for more advanced services of communication, providing high speed internet and content delivery in networks. 
- PCRF: the PCRF instructs the network about the Qos and the rules of the policies for charging based on the information extracted from the repository of the subscriber. It is also responsible for decision-making for the policy control.

- Mobile Management Entity (MME): the MME is in charge of preparing the signs between the center system and the client gadget or UE. It additionally incorporates association administration like setting up association among system and UE and conveyor administration, for example, discharge, foundation and upkeep.

- HSS: the HSS contains information regarding the UE connected to the network. It provides authorization, identification and provisioned services to the user.

\section{ADVANTAGES}

There are some advantages of using the 4G LTE network which makes it the best among all other network. These are:

- It provides high-quality video or voice services over the LTE network

- Quick and easy deployment of services over WIFI/LTE

- It has a fast time-to-market [4] strategy and has full integration with the existing network

- Core elements are able to run on the same old existing platform reducing the ownership cost

- It provides better services to the mobile phone users at a cheaper rate

- The operator gets more revenue [3] from the prioritized delivery of data packets over the network [12]

- It provides professional services for planning, design and integration

- Provides full mobility and voice service continuity in future

\section{DISADVANTAGES}

There are some technical and non-technical reasons due to which many people and the operators will not adopt VoLTE very soon.

Technical Drawbacks: there are two main features that are not satisfied by VoLTE. First is the energy saving mechanism that has a fixed packet delivery time fixed for all the packets that transfer over the same VoIP communication network, due to this variation in packet size the energy consumption is high in VoLTE. The second is VoLTE does not have coverage enhancement mechanism in which Transmission Time Interval (TTI) [13] is deployed. The VoLTE transfers many duplicate voice packets in a time slot consecutively. According to the reports [14] the VoLTE can upgrade the coverage by 2-4 dB.

Non-technical Drawbacks: the VoLTE requires the service providers and the carriers to collaborate their resources. But this may lead to sharing of the revenue collected from the subscribers among the carrier and the provider which may not benefit the business of a single person. The carrier always have to ask or discuss their business plans with the service provider every time and it may not convince the other party or benefit them which may cause rejection of newly made plans which are highly recommended by the customers and in return bring loss in business of the carriers.

VIII. CONCLUSION 
In our paper we show that the abilities of VoLTE make it possible to deploy in $4 \mathrm{G}$ or not. It also plots the graph between cost of deployment and processes [16] as well as the advantages provided to different parties. We have seen that now-a-days many service providers are taking interest in deploying VoLTE services for the customers and have grown their market value at the highest like the $\mathrm{JIO} 4 \mathrm{G}$ services from the reliance groups. As messaging, video and voice quality has become the necessity of day to day life so, VoLTE gives $4 \mathrm{G}$ the ability to provide these services. VoLTE helps to expand the limitations of voice calling facilities in the near future. The IMS subsystem does not provide priority services as provided by LTE thus, removing the biggest hurdle that may lead to fast rollout from the market.

\section{REFERENCES}

[1] Voice over LTE. http://www.gsma.com/technicalprojects/volte.

[2] 3GPP. TS23.228: IP Multimedia Subsystem (IMS);Stage 2, 2014.

[3] Volte market revenue share of $12 \%$ to be held by video calling and supplementary services in 2020 , Oct 2015. http://www.prnewswire.com/news-releases/volte-market-revenueshare-of-12-to-be-held-by-video-calling-and-supplementaryservicesin-2020-530219051.html.

[4] AT\&T: 2015 will be the year of mass market VoLTE. http://www.fiercewireless.com.

[5] RFC3261: SIP: Session Initiation Protocol, June 2002.

[6] RFC 3550: RTP: A Transport Protocol for Real-Time Applications,Jul 2003.

[7] 3GPP. Operation, Adminstration, Maintenance (OAM).

[8] 3GPP. TS 23.216: Single Radio Voice Call Continuity (SRVCC),March 2011.

[9] P.800: Methods for subjective determination of transmission quality.

[10] Audacity. http://audacity.sourceforge.net/.

[11] 3GPP. TS24.301: Non-Access-Stratum (NAS) protocol for Evolved Packet System (EPS); Stage 3, Jun. 2013.

[12] Experian: Americans spend 58 minutes a day on their smartphones. http://www.experian.com.

[13] 3GPP. TR36.824: LTE coverage enhancements, 2012.

[15] N. Networks. From Voice over IP to Voice over LTE, 2013. http://tinyurl.com/q79vyu6.

[15] WebRTC web site.http://www.webrtc.org

[16] White Paper: Troubleshooting LTE and VoLTE service issues for Mobile Operators. 